\title{
AN ENERGETIC VARIATIONAL FORMULATION WITH PHASE FIELD METHODS FOR INTERFACIAL DYNAMICS OF COMPLEX FLUIDS: ADVANTAGES AND CHALLENGES
}

\author{
JAMES J. FENG*, CHUN LIU ${ }^{\dagger}$, JIE SHEN ${ }^{\ddagger}$, AND PENGTAO YUE $\S$
}

\begin{abstract}
The use of a phase field to describe interfacial phenomena has a long and fruitful tradition. There are two key ingredients to the method: the transformation of Lagrangian description of geometric motions to Eulerian description framework, and the employment of the energetic variational procedure to derive the coupled systems. Several groups have used this theoretical framework to approximate Navier-Stokes systems for two-phase flows. Recently, we have adapted the method to simulate interfacial dynamics in blends of microstructured complex fluids. This review has two objectives. The first is to give a more or less self-contained exposition of the method. We will briefly review the literature, present the governing equations and discuss a numerical scheme based on different numerical schemes, such as spectral methods. The second objective is to elucidate the subtleties of the model that need to be handled properly for certain applications. These points, rarely discussed in the literature, are essential for a realistic representation of the physics and a successful numerical implementation. The advantages and limitations of the method will be illustrated by numerical examples. We hope that this review will encourage readers whose applications may potentially benefit from a similar approach to explore it further.
\end{abstract}

Key words. Energetic variational formulation, phase field methods, Cahn-Hilliard equation, two-phase flows, complex fluids, free interfacial motions.

AMS(MOS) subject classifications. 76A02, 76A15, 76A05, 76M30, 76T20, 76T10, 76R99, 76M45,76M22, 76D45, 76B10, 76D05

1. Introduction. Most complex fluids have complicated internal microstructures, whose conformation is coupled with the macroscopic dynamics of the material [1]. On the one hand, this coupling gives rise to novel flow behavior. On the other, it plays a central role in achieving desirable

*Department of Chemical \& Biological Engineering and Department of Mathematics, University of British Columbia, Vancouver, BC V6T 1Z4, CANADA (jfeng@chml.ubc.ca).

${ }^{\dagger}$ Department of Mathematics, Penn State University, University Park, PA 16802 (liu@math.psu.edu).

‡Department of Mathematics, Purdue University, West Lafayette, IN 47907 (shen@math.purdue.edu).

$\S$ Department of Chemical \& Biological Engineering and Department of Mathematics, University of British Columbia, Vancouver, BC V6T 1Z4, CANADA. 
structure and property in advanced engineering materials. Complex fluids are often used in composites, of which polymer-dispersed liquid crystals and polymer blends are good examples $[2,3]$. In these two-phase systems, the components are separated by myriad interfaces that move and deform with the flow; the interfacial morphology to a large extent determines the dynamics of the mixture.

A fluid-mechanical theory for two-phase mixtures of complex fluids has to contend with two difficulties: the moving internal boundaries (or internal transition regions) and the complex rheology of the components. The former is a well-known mathematical difficulty. The movement of the interfaces is naturally amenable to a Lagrangian description, while the bulk flow is conventionally solved in an Eulerian framework. A great deal of effort has gone to reconciling these two considerations in a numerical scheme [4]. The latter difficulty stems from the fact that the rheology of each component depends on the internal microstructure, which is coupled with the flow field $[5$, e.g.]. Thus, these materials feature dynamic coupling of three disparate length scales: molecular or supra-molecular conformation inside each component, mesoscopic interfacial morphology and macroscopic hydrodynamics. The complexity of such materials has for the large part prohibited theoretical and numerical analysis.

A conceptually straightforward way of handling the moving interfaces is to employ a moving mesh that has grid points on the interfaces and deforms according to the flow on both sides of the boundary. This has been implemented in boundary integral and boundary element methods [6-8], finite-element methods [9-11] and a finite-difference method [12,13]. Besides the overhead in keeping track of the moving mesh, these methods break down when large displacement of internal domains causes mesh entanglement or when the interfaces undergo singular topological changes such as breakup and coalescence. Thus, these methods have been limited mostly to single drops undergoing relatively mild deformations. As an alternative, fixed-grid methods have been developed that regularized the interface [4]. These include the volume-of-fluid (VOF) method [14,15], the front-tracking method $[16,17]$ and the level-set method [18-20]. All these approaches have the advantage of converting the Lagrangian description 
of a geometric motion into the Eulerian description. Instead of computing the flow of the two components with matching boundary conditions on the interface, these methods represent the interfacial tension as a body force or bulk stress spread over a narrow region covering the interface. Then a single set of governing equations can be written over the entire domain and solved on a fixed grid in a purely Eulerian framework.

The phase-field method is also a fixed-grid method; it differs from those aforementioned in that the interface is diffuse in a physical rather than numerical sense. Thus, it is also known as the diffuse-interface model. More precisely, the diffuse interface is introduced through an energetic variational procedure that results in a thermodynamic consistent coupling system. The basic idea was derived from the consideration that the two components, though nominally immiscible, does mix in reality within a narrow interfacial region. A phase-field variable $\phi$ is introduced, which can be thought of as the volume fraction, to demarcate the two species and indicate the location of the interface. A mixing energy is defined based on $\phi$ which, through a convection-diffusion equation, governs the evolution of the interfacial profile. The phase-field method can be viewed as a physically motivated level-set method, and Lowengrub and Truskinovsky [21] have argued for the advantage of using a physically determined $\phi$ profile instead of an artificial smoothing function for the interface. When the thickness of the interface approaches zero, the diffuse-interface model becomes asymptotically identical to a sharp-interface level-set formulation. It also reduces properly to the classical sharp-interface model in general.

The idea of diffuse interfaces can be traced back to van der Waals [22-25], and has since been developed for numerous applications, e.g., phase transition and critical phenomena [26,27], solidification and dendritic growth in alloys $[28,29]$, interfacial tension theories [30], phaseseparation $[27,31,32]$ and two-phase flows [33-40]. Recently, Yue et al. [41] has generalized the theoretical model to simulate interfacial dynamics in complex fluids. Taking advantage of the energy-based formulation, they are able to resolve the dual difficulties for complex fluid mixtures - moving interfaces and complex rheology —in a unified framework. So far, Yue and coworkers have applied the method to a number of problems on drop dynamics of viscoelastic and liquid crystalline fluids [42-46]. In the following, we first give a brief but self-contained derivation of the theoretical model, and describe a numerical algorithm using spectral methods. Then we will illustrate the advantages and limitations of the model by numerical examples. We hope to convince the reader that the diffuse-interface idea can be developed into a unique CFD tool for multi-phase and multi-component complex fluids. 
2. An energy-based phase-field theory. The phase-field model can be derived from the general procedure of Lagrangian mechanics [21,37]. We write out the Lagrangian (action functional) of the system based on its free energy, and carry out variations with respect to the field variations (and the flow map). This amounts to following the "least-action principle" and various dynamical laws, and will lead to evolution equations for these variables (including the momentum equation - force balance equations). The dissipative portions of these equations need to be derived separately, for instance via irreversible thermodynamics [47]. The entire procedure has been demonstrated previously for Newtonian, viscoelastic and nematic liquid-crystalline fluids [37,41,48], and even for fluid-structure interactions (with the help of a Eulerian description of elasticity) [49]. In the following, we will use an example of a Newtonian-nematic blend with planar anchoring for illustration.

For an immiscible blend of a nematic liquid crystal and a Newtonian fluid, there are three types of free energies: mixing energy of the interface, bulk distortion energy of the nematic, and the anchoring energy of the liquid crystal molecules on the interface. We introduce a phase-field variable $\phi$ such that the concentration of the two components is $(1+\phi) / 2$ and $(1-\phi) / 2$, respectively. We express the mixing energy density in the Landau-Ginzburg form:

$$
f_{\text {mix }}(\phi, \nabla \phi)=\frac{\lambda}{2}|\nabla \phi|^{2}+\frac{\lambda}{4 \epsilon^{2}}\left(\phi^{2}-1\right)^{2},
$$

where the parameter $\lambda$ is the mixing energy density, and $\epsilon$ is a capillary width representative of the thickness of interface. The gradient energy term $\lambda|\nabla \phi|^{2} / 2$ and the bulk energy term $f_{0}=\lambda\left(\phi^{2}-1\right)^{2} /\left(4 \epsilon^{2}\right)$ represent the "philic" and "phobic" tendencies between the species, their competition giving rise to the equilibrium $\phi$ profile. Note that $f_{m i x}$ is the diffuseinterface counterpart of the interfacial tension. In fact, one can relate the conventional interfacial tension $\sigma$ to the parameters in the mixing energy. For instance, from an equilibrium hyperbolic-tangent $\phi$ profile that is the 1D energy minimizer, one obtains $[34,41]$

$$
\sigma=\frac{2 \sqrt{2}}{3} \frac{\lambda}{\epsilon}
$$


The orientation of the nematic liquid crystal is described by the director field $\boldsymbol{n}(\boldsymbol{x})$. The Frank distortion energy expresses the energy penalty for distorting the orientation [50]:

$$
f_{\text {bulk }}=K\left[\frac{1}{2} \nabla \boldsymbol{n}:(\nabla \boldsymbol{n})^{\mathrm{T}}+\frac{\left(|\boldsymbol{n}|^{2}-1\right)^{2}}{4 \delta^{2}}\right],
$$

where $K$ is the elastic constant. The second term on the right-hand side regularized the original Frank energy to allow defects [51]. The nematic prefers to orient on the interface along an easy axis [50]; any deviation from it is penalized by an anchoring energy. Here we assume that the easy axis is any direction in the tangential plane, and write the anchoring energy as

$$
f_{\text {anch }}=\frac{A}{2}(\boldsymbol{n} \cdot \nabla \phi)^{2},
$$

with the positive constant $A$ representing the anchoring strength. This is the diffuse-interface counterpart of the Rapini-Popoular energy [52]. Unlike in the sharp-interface picture, both $f_{m i x}$ and $f_{\text {anch }}$ are volumetric energy densities. Finally, the total free energy density for the two-phase material is written as:

$$
f(\phi, \boldsymbol{n}, \nabla \phi, \nabla \boldsymbol{n})=f_{m i x}+\frac{1+\phi}{2} f_{\text {bulk }}+f_{\text {anch }}
$$

where $(1+\phi) / 2$ is the volume fraction of the nematic component, and $\phi=1$ in the purely nematic phase.

Variation of the system's action functional with respect to the phasefield variable $\phi$, the nematic director $\boldsymbol{n}$ and the displacement leads to evolution equations for $\phi, \boldsymbol{n}$ and the momentum equation. Augmented by the dissipative effects, the governing equations of the system are:

$$
\begin{aligned}
\frac{\partial \phi}{\partial t}+\boldsymbol{v} \cdot \nabla \phi & =\gamma_{1} \nabla^{2} \frac{\delta F}{\delta \phi} \\
\frac{\partial \boldsymbol{n}}{\partial t}+\boldsymbol{v} \cdot \nabla \boldsymbol{n} & =\gamma_{2} \boldsymbol{h}, \\
\nabla \cdot \boldsymbol{v} & =0, \\
\rho\left(\frac{\partial \boldsymbol{v}}{\partial t}+\boldsymbol{v} \cdot \nabla \boldsymbol{v}\right) & =-\nabla p+\nabla \cdot\left[\mu\left(\nabla \boldsymbol{v}+\nabla \boldsymbol{v}^{T}\right)+\boldsymbol{\sigma}^{e}\right],
\end{aligned}
$$


where $\gamma_{1}$ is the interfacial mobility and $\gamma_{2}$ determines the relaxation time of $\boldsymbol{n} . F=\int f d \Omega$ is the total free energy of the system, whose variations produce

$$
\frac{\delta F}{\delta \phi}=\lambda\left[-\nabla^{2} \phi+\frac{\phi\left(\phi^{2}-1\right)}{\epsilon^{2}}\right]+\frac{1}{2} f_{b u l k}-A \nabla \cdot[(\boldsymbol{n} \cdot \nabla \phi) \boldsymbol{n}],
$$

and the molecular field

$$
\boldsymbol{h}=-\frac{\delta F}{\delta \boldsymbol{n}}=K\left[-\nabla \cdot\left(\frac{1+\phi}{2} \nabla \boldsymbol{n}\right)+\frac{1+\phi}{2} \frac{\left(\boldsymbol{n}^{2}-1\right) \boldsymbol{n}}{\delta^{2}}\right]+A(\boldsymbol{n} \cdot \nabla \phi) \nabla \phi .
$$

Note that the right-hand side of the dynamic equation (2.6) dictates the relaxation of the phase-field variable $\phi$, with a relaxation time proportional to $1 / \gamma_{1}$. In the limit of $\gamma_{1}$ approaching zero, we recover the kinematic condition for the interface. Moreover, as $\epsilon$ approaches zero, the dynamics of $\phi$ will preserve the profile of the transition (hyperbolic-tangent in this case), a key advantage of phase field approach. The last two terms in equation (2.10) represent coupling between the phase field and the Frank distortion energy and anchoring energy. When the interface is thin, $f_{\text {bulk }}$ is dominated by the mixing energy near the interface and therefore negligible. The last term may have an effect on the interfacial $\phi$ profile for strong anchoring. But it is a higher order effect, negligible if the effects of interfacial tension and surface anchoring are assumed to be additive (cf. equation (2.12) below). Thus for simplicity, the last two terms on the right-hand-side of equation (2.10) are neglected hereafter. There are applications, e.g. [28], where the interface is relatively thick and the $\phi$ profile has physical consequences.

In the variation with respect to displacement, we have assumed equal density between the two species. A small density mismatch may be handled by the Boussinesq approximation [37]. In the more general situation, the mass-averaged mixture velocity becomes non-solenoidal within the interfacial region, and a theory for compressible mixtures can be constructed [21]. The pressure is a Lagrange multiplier for the constraint of incompressibility. The elastic stress tensor is derived as part of the variational procedure [41], and in this case can be written out as

$$
\boldsymbol{\sigma}^{e}=-\lambda(\nabla \phi \otimes \nabla \phi)-K \frac{1+\phi}{2}(\nabla \boldsymbol{n}) \cdot(\nabla \boldsymbol{n})^{\mathrm{T}}-A(\boldsymbol{n} \cdot \nabla \phi) \boldsymbol{n} \otimes \nabla \phi .
$$


3. Numerical scheme. While the coupled nonlinear system $\left(2.6^{-}\right.$ 2.9) are adequate mathematical models for the mixtures of complex fluids, it is a challenging task to construct a numerical scheme which is capable of correctly capturing, at a reasonable cost, the complex spatial and temporal features of these two-phase flows.

We propose to discretize the coupled nonlinear system (2.6-2.9) in time with a stabilized semi-implicit second-order scheme. The guiding principle here is that we only want to solve decoupled, constant-coefficient elliptic equations at each time step while preserving the overall second-order time accuracy and having a reasonably large stability region.

To simplify the presentation, we shall only describe our approach for the Cahn-Hilliard equation

$$
\frac{\partial \phi}{\partial t}+\gamma \nabla^{2}\left(\nabla^{2} \phi-\frac{\left(|\phi|^{2}-1\right) \phi}{\epsilon^{2}}\right)=h_{1},
$$

and for the time-dependent Stokes equations

$$
\begin{aligned}
& \frac{\partial \boldsymbol{v}}{\partial t}-\nu \Delta \boldsymbol{v}+\nabla p=\boldsymbol{h}_{\mathbf{2}}, \\
& \nabla \cdot \boldsymbol{v}=0,
\end{aligned}
$$

where the forcing functions $h_{1}$ and $\boldsymbol{h}_{\mathbf{2}}$ would include all the extra nonlinear terms in (2.6-2.9) which will be treated explicitly to avoid solving nonlinear equations at each time step. The treatment for the nematic director equation 2.7) is very similar.

Let us consider first the Cahn-Hilliard equation (3.1). A main difficulty associated with the numerical approximation of (3.1) is that a standard semi-implicit scheme leads to a very stiff system (when $\epsilon \ll 1$ ) which dictates a very small time step. This difficulty can be alleviated by using the following shifted semi-implicit scheme:

$$
\begin{aligned}
& \frac{3 \phi^{k+1}-4 \phi^{k}+\phi^{k-1}}{2 \Delta t}+\gamma\left(\Delta^{2}-\frac{C_{s}}{\epsilon^{2}} \Delta\right) \phi^{k+1}=2 h_{1}^{k}-h_{1}^{k-1} \\
&+\frac{\gamma}{\epsilon^{2}} \Delta\left[2\left(\left|\phi^{k}\right|^{2}-\left(1+C_{s}\right)\right) \phi^{k}\right. \\
&\left.-\left(\left|\phi^{k-1}\right|^{2}-\left(1+C_{s}\right)\right) \phi^{k-1}\right] ; \\
& 7
\end{aligned}
$$


where $C_{s}$ is a stabilizing parameter typically in the range of $[1,5]$. Ample numerical results indicate that the above stabilized semi-implicit scheme allows much larger time step than the standard semi-implicit scheme does. We observe that (3.3) is a fourth-order equation for $\phi^{k+1}$ with constant coefficients.

Next, we describe our approach for solving the time-dependent Stokes problem (3.2).

- If the boundary conditions are periodic, the pressure in (3.2) can be easily eliminated using the divergence-free conditions so (3.2) can be efficiently solved by using a Fourier-spectral method [37].

- If the velocity satisfies a free-slip boundary condition (cf. [53]), then, the time discrete approximation of (3.2) can be split into a sequence of Poisson-type equations for the velocity and for the pressure.

- If the boundary conditions in all but one direction are periodic, (3.2) can be reduced into a sequence of one-dimensional fourthorder equations using a Fourier expansion in all but one direction [54].

- Finally, for the general cases, we shall use a projection type scheme (see the recent review paper [55]) to decouple the computation of the velocity from the pressure. For example, we may use the new consistent splitting scheme introduced in [56]. To be specific, we assume here that the velocity is subjected to a homogeneous Dirichlet boundary condition:

$$
\begin{gathered}
\frac{3 \boldsymbol{v}^{k+1}-4 \boldsymbol{v}^{k}+\boldsymbol{v}^{k-1}}{2 \Delta t}-\nu \Delta \boldsymbol{v}^{k+1}+\nabla\left(2 p^{k}-p^{k-1}\right) \\
=2 \boldsymbol{h}_{\mathbf{2}}{ }^{k}-\boldsymbol{h}_{\mathbf{2}}{ }^{k-1}, \\
\left.\boldsymbol{v}^{k+1}\right|_{\partial \Omega}=0, \\
\left(\nabla \psi^{k+1}, \nabla q\right)=\left(\frac{3 \boldsymbol{v}^{k+1}-4 \boldsymbol{v}^{k}+\boldsymbol{v}^{k-1}}{2 \Delta t}, \nabla q\right), \forall q \in H^{1}(\Omega), \\
p^{k+1}=\psi^{k+1}+2 p^{k}-p^{k-1}-\nu \nabla \cdot \boldsymbol{v}^{k+1},
\end{gathered}
$$

Note that (3.4) is a Poisson-type equation for $\boldsymbol{v}^{k+1}$ while (3.5) is a Poisson equation (with homogeneous Neumann boundary conditions) in the weak form for $\psi^{k+1}$. 
Hence, after a time discretization to the coupled nonlinear system (2.6-2.9), we only need to solve, at each time step, a sequence of constantcoefficient elliptic equations which can be efficiently handled by one of the many existing numerical methods using finite difference, finite elements or spectral methods. Since we shall confine ourselves to simple geometries in this study, we choose to use the well-conditioned and fast spectral-Galerkin methods developed in $[54,57,58]$ which are capable of solving constantcoefficient elliptic equations in simple geometries with quasi-optimal computational complexity, i.e., the number of operations per time step is of order $O(N \log N), N$ being the number of unknowns. The high resolution property of the spectral method and the efficiency of the fast spectralGalerkin algorithms allow us to numerically solve the coupled nonlinear system (2.6-2.9) at a reasonable cost. For example, with a $750 \mathrm{MHz}$ Sparcv9 processor, the two-dimensional problems with a spatial resolution of $1024 \times 1024$ or $2048 \times 1024$ typically take about 1 minute of CPU time per time step. For all the simulations reported below, we have carried out grid and time-step refinements to ensure convergence. If we take $4.164 \epsilon$ to be a nominal interfacial thickness (cf. [41]), this layer typically requires 7-10 grids to resolve. Coarser grids will generate spurious oscillations in the solution, especially in the vicinity of the interface.

4. Advantages of the diffuse-interface model. Needless to say, the greatest payoff of adopting a diffuse-interface picture is the ease with which moving interfaces can be handled. Compared with the traditional sharp-interface view of internal boundaries, there is no longer a need to track the position of the interface, and to impose matching boundary conditions for solving the flow inside each component separately. As mentioned earlier, the interfacial tension is now represented by an elastic stress tensor concentrated in the interfacial region. The cost is the additional dynamics for $\phi$; we have to deal with the physics of the convection-diffusion process as well as the numerical burdens of an additional equation. These will be discussed in the next section among the subtle issues that need special consideration.

The diffuse-interface formulation also brings about several "side benefits" that may be of great importance to the physical applications at hand. Here, we illustrate in some detail three of such benefits that we have noted in our simulations. These advantages reflect the fact that the phase-field idea transforms the Lagrangian description of a geometric motion into Eulerian coordinates, and easily represents the competition between various energy functionals for the multiphase material. 


\subsection{Short-range molecular forces during topological changes.}

For the same reason that the phase-field method handles moving interfaces easily, so it does singular topological changes such as breakup and coalescence. In the sharp-interface convention, such events require an ad hoc treatment. For filament breakup and drop coalescence, for example $[59,60]$, the thinning neck or film has to be artificially removed once its thickness reaches a prescribed threshold. In contrast, the diffuse-interface is represented by the contour of $\phi=0$, which deforms and reconnects smoothly during flow. Thus, no artificial trigger is needed for drop breakup and coalescence. As an example, Fig. 1 illustrates the head-on collision and subsequent coalescence of two Newtonian drops in a Newtonian matrix. The draining film develops a "dimple" in the middle [61] and the rupture occurs toward the outside of the film, trapping some matrix fluid inside.

In reality, film rupture is effected by short-range forces such as van der Waals force [62]. Interestingly, the phase-field model is rooted in the physics of molecular interaction between the two species, and thus contains short-range molecular forces. To see this, consider the simple situation in Fig. 2, with a liquid film (F) of uniform thickness $h$ sandwiched between semi-infinite domains of another fluid (A). For a thick film, the phase-field variable at the center approaches the bulk value, say $\phi_{0} \rightarrow-1$, at the center. For a thin film, however, conceivably $\phi$ inside $\mathrm{F}$ will differ from the bulk value: $\phi_{0}>-1$. From the elastic stress tensor due to the mixing energy (cf. [42]), one may calculate the disjoining pressure in the diffuseinterface model:

$$
\Pi_{\phi}=-\lambda f_{0}=-\frac{\lambda\left(\phi_{0}^{2}-1\right)^{2}}{4 \epsilon^{2}} .
$$

which implies an attractive force between the interfaces as with van der Waals force. If we estimate $\phi_{0}$ based on a hyperbolic tangent $\phi$-profile as in a one-dimensional equilibrium interface [41],

$$
\phi_{0}=-\tanh \left(\frac{h}{2 \sqrt{2} \epsilon}\right),
$$

Then the disjoining pressure in Eq. (4.1) can be shown to be of the same order of magnitude as the van der Waals force. As the film thickness approaches zero, however, the van der Waals force goes to infinity while $\Pi_{\phi}$ remains finite. A more detailed comparison can be found in Ref. [42]. On a fundamental level, the discrepancy between van der Waals force and $\Pi_{\phi}$ stems from the truncation of the Cahn-Hilliard free energy at the quadratic term $|\nabla \phi|^{2}$. An elegant explanation has been given by Pismen [63]. 

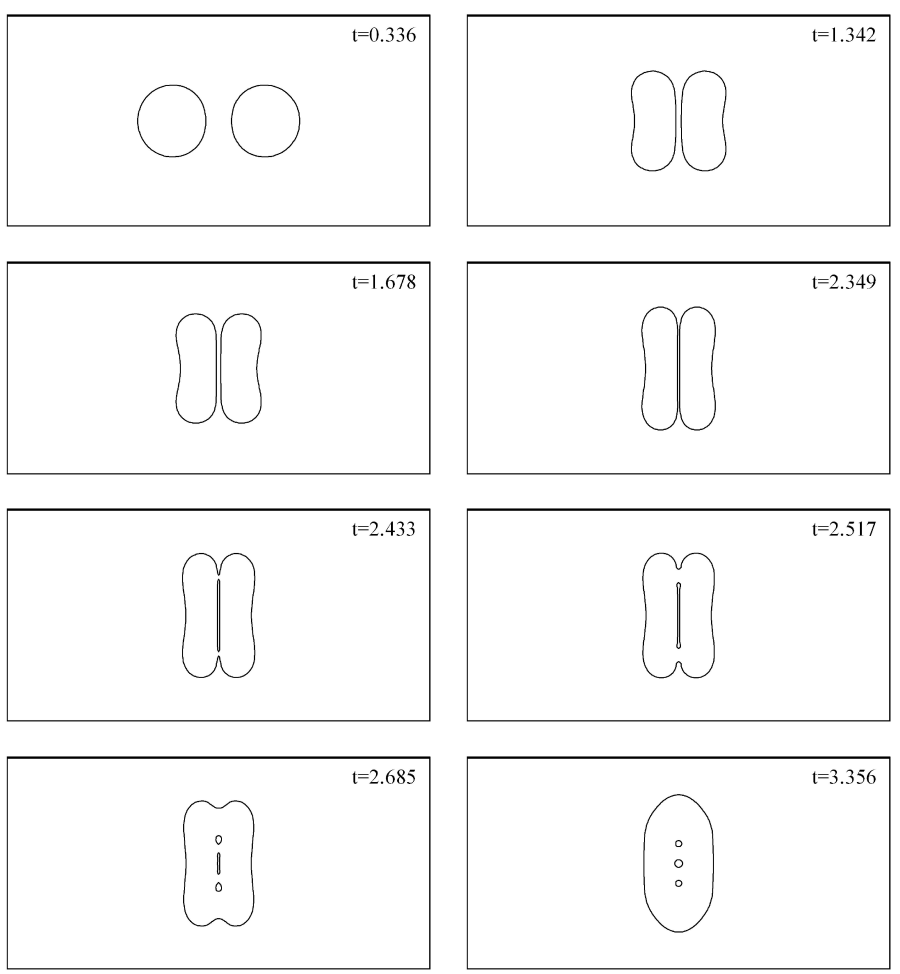

Fig. 1. Collision and coalescence of two Newtonian drops in a Newtonian matrix. The Reynolds number, defined using $D$ and $U$, is Re $=33.6$, and the Weber number is $W e=12$. Other parameters are: $\epsilon=0.01$ and $\gamma=3.365 \times 10^{-5}$ (after Yue et al. [41], (c) Cambridge University Press.)

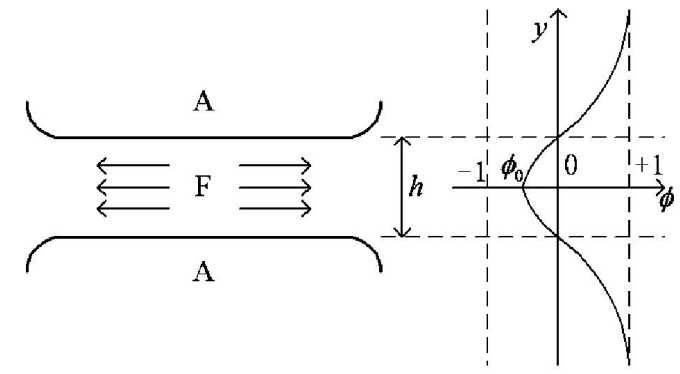

FIG. 2. A cartoon for a draining film and the corresponding $\phi$ profile.

4.2. Complex rheology. Because of its energy-based formalism, our diffusive interface method incorporates complex rheology easily. The nonNewtonian rheology is typically due to microstructures whose conformation 
deviates from equilibrium under deformation. The conformation of the microstructure is often governed by a free energy, e.g., the Frank distortion energy for a liquid crystal or the free energy of a polymer chain. In Section 2, we showed how this microstructural energy can be added to the mixing energy to form the total free energy of the multi-phase system, which will give rise to the proper constitutive equation for the microstructured fluids in addition to the evolution equation of the phase field variable. Thus, interfacial dynamics and complex rheology are included in a unified theoretical framework.

This procedure is general in that various types of constitutive relations can be derived by the same procedure. As a second example, we consider here the important case of a viscoelastic polymer solution modeled as a suspension of Hookean dumbbells in a Newtonian solvent [64]. Instead of the least-action principle, we follow a formally different but essentially equivalent "virtual-work principle" [5]. For a single dumbbell with a connector $\boldsymbol{Q}$, its elastic energy is $\frac{1}{2} H \boldsymbol{Q} \cdot \boldsymbol{Q}$, where $H$ is the elastic constant. For an ensemble of dumbbells with configuration distribution $\Psi(\boldsymbol{Q})$, the average energy can be written as

$$
f_{d}=\int_{R^{3}}\left(k T \ln \Psi+\frac{1}{2} H \boldsymbol{Q} \cdot \boldsymbol{Q}\right) \Psi d Q,
$$

where $k$ is the Boltzmann constant and $T$ is the temperature, and the integration is over all possible configurations of $\boldsymbol{Q}$. Now the total free energy density of the two-phase system is:

$$
f=f_{m i x}+\frac{1+\phi}{2} n f_{d}
$$

where $n$ is the number density of the dumbbells. Since the stress tensor due to $f_{m i x}$ has been derived (cf. equation 2.12 and [41]), we will only consider the elastic stress due to the dumbbell energy $f_{d}$. We impose a virtual displacement $\delta \boldsymbol{x}$ on the material, which takes place instantaneously so that the dumbbells deform affinely with no slip between the bead and the surrounding fluid. The corresponding change in the distribution function $\Psi$ can be obtained from the Fokker-Planck equation for $\Psi$ [64]. Now we may 
calculate the resultant variation in the dumbbell free energy. Omitting the intermediate steps [42], we eventually arrive at:

$$
\begin{aligned}
\delta f_{d} & =\int_{R^{3}}\left(k T \ln \Psi+k T+\frac{H}{2} \boldsymbol{Q}: \boldsymbol{Q}\right) \delta \Psi d Q \\
& =(-k T \boldsymbol{I}+H<\boldsymbol{Q} \boldsymbol{Q}>):(\nabla \delta \boldsymbol{x})^{\mathrm{T}},
\end{aligned}
$$

where $<\cdot\rangle=\int_{R^{3}} \cdot \Psi d Q$ and $\boldsymbol{I}$ is the identity tensor. Thus the dumbbell stress tensor is:

$$
\boldsymbol{\tau}_{d}=-n k T \boldsymbol{I}+n H<\boldsymbol{Q Q}>
$$

which obeys the Maxwell equation. This is exactly the Kramers expression for the polymer elastic stress tensor [64]. The same procedure can be followed for other microstructural free energies, such as the Marrucci-Greco nematic potential energy for liquid-crystalline polymers $[5,65]$.

4.3. Energy conservation. An additional advantage of the phasefield method over other interface-regularizing methods is its energy conservation: a solution to the governing equations in Section 2 obeys an energy law. For example, multiplying equation (2.9) by the velocity $\boldsymbol{v}$, equation (2.6) by the chemical potential $\delta F / \delta \phi$ and equation (2.7) by the molecular field $\delta F / \delta \boldsymbol{n}$, integrating over the entire domain and summing the results, we obtain:

$$
\frac{d}{d t} \int_{\Omega}\left(\frac{\rho}{2}|\boldsymbol{v}|^{2}+f\right) d \Omega=-\int_{\Omega}\left(\mu \nabla \boldsymbol{v}: \nabla \boldsymbol{v}^{\mathrm{T}}+\gamma_{1}\left|\nabla \frac{\delta F}{\delta \phi}\right|^{2}+\gamma_{2}\left|\frac{\delta F}{\delta \boldsymbol{n}}\right|^{2}\right) d \Omega,
$$

where $f$ is the system's potential energy density (cf. equation 2.5), and surface work has been omitted. Physically, the law states that the total energy of the system (excluding thermal energy) will decrease from internal dissipation. Based on such energy laws, Lin and Liu [66,67] have established the existence of classical and weak solutions for Leslie-Ericksen fluids. In general, energy laws play an important role in the convergence of finite-dimensional approximations to partial differential equations, especially when the solution is not smooth [51]. This constitutes one of the advantages of our method over previous methods that do not maintain the system's total energy budget. In VOF simulations, density is the labeling 
function subjected to smoothing. The level-set method renormalizes the distance function. In either case, the conservation of energy cannot be maintained.

Note that the energy conservation holds exactly when all the coupling terms in equation (2.10) are kept. For numerical conveniences, we have omitted such terms in applications where the interface will remain thin and the coupling terms have at most a localized effect. This omission will violate the energy conservation. When the geometry is simple and the solution is smooth, non-conservation of energy usually does not compromise the quality of the solution. But difficulties may arise in the presence of rapid spatial variations, which are characteristic of microstructured fluids with internal boundaries and/or defects $[1,43]$.

5. Physical and numerical subtleties. Although the convergence of the phase-field model to the sharp-interface model has been established by asymptotic expansion for regular velocity fields [21, 24, 25, 33, 37, 39, 40], there are some subtle issues that merit further discussion. One such issue, for example, concerns incompressibility. While the phase-field formulation imposes incompressibility throughout the domain (hence also on the interface), the sharp-interface model satisfies this condition only weakly on the interface. In fact, the system would be over-determined with such a constraint on the interface. For phase-field models, we are allowed to impose $\nabla \cdot \boldsymbol{v}=0$ everywhere thanks to the diffused transition layer. The same holds for VOF and level-set methods through the introduction of an artificial transition layer. Physically, one may consider the sharp interface and the diffuse interface different approximations of the real physical situation, the former by relaxing incompressibility on the interface and the latter by introducing the transition layer.

The phase-field method can be viewed from two complementary angles: as a representation of the microscopic physics on the interface or as a numerical device for simulating moving boundary problems without tracking the interface. Depending on the applications, one or the other viewpoint may be more appropriate. For applications such as solidification of alloys $[28,29]$ and near-critical systems $[26,33]$, it is essential to ensure 
that the phase-field equation captures the dynamics at the interface because the interfacial profile is of direct interest. On the other hand, the two-phase flow problems we have simulated involve "immiscible" components with interfacial thickness on the order of tens of nanometers. Beyond indicating the position and movement of the interface, the $\phi$ profile has little direct bearing on the macroscopic properties of interest. Thus, there is a degree of freedom or ambiguity in choosing the dynamics of the phase field and the parameter values. In particular, the interfacial thickness in the model can be much thicker than in reality; there is no need, nor perhaps the capability, to resolve the interface down to nanometer scales. From this an array of subtle issues arise, which must be handled with care for the model to be physically sound and numerically efficient.

5.1. Cahn-Hilliard and Allen-Cahn dynamics. As long as our physical problem conceptually consists of sharp interfaces, the diffusion dynamics of the phase-field variable is to a large extent fictitious. Thus, one can choose Cahn-Hilliard, Allen-Cahn or other types of dynamics. We can view all such choices as a relaxation or approximation of the kinematic transport equations. Based on similar considerations, we have neglected certain coupling terms in the Cahn-Hilliard equation due to presence of microstructures (cf. equation 2.10). One requirement on the diffusion dynamics is that they maintain the integrity of the interface. In other words, the "phobic" and "philic" tendencies should be balanced such that the transition layer neither smoothes out nor steepens into a shock wave.

The Cahn-Hilliard equation follows from the physical argument that the flux be proportional to the gradient of a generalized chemical potential. This differs from the conventional Fick's law, which leads to the Allen-Cahn dynamics. The advantage of the Cahn-Hilliard equation is the following conservation of total system "mass":

$$
\frac{d}{d t} \int_{\Omega} \phi(x, t) d x=0
$$

if the following no-flux boundary condition is imposed.

$$
\frac{\partial}{\partial \boldsymbol{n}}\left(\frac{\delta F_{m i x}}{\delta \phi}\right)=0,
$$


where $\boldsymbol{n}$ is the normal direction to the boundary.

A disadvantage of the Cahn-Hilliard equation is that its higher (4th) order causes numerical complications. Shen [54] and Yue et al. [41] used a procedure of splitting it into two second-order Helmholtz equations.

The Allen-Cahn equation is easier to handle numerically but does not automatically ensure conservation of mass; a Lagrange multiplier can be introduce to enforce it as a constraint [68]:

$$
\frac{\partial \phi}{\partial t}+\boldsymbol{v} \cdot \nabla \phi=\gamma_{1}\left(-\frac{\delta F}{\delta \phi}+\sigma\right),
$$

with $\int_{\Omega} \phi(x, t) d x=\int_{\Omega} \phi(x, 0) d x$.

Another possibility is the "advected field" method [69], which is a compromise between phase-field and level-set approaches. To impose mass conservation on the Allen-Cahn equation, an additional term proportional to the local curvature is added:

$$
\frac{\partial \phi}{\partial t}+\boldsymbol{v} \cdot \nabla \phi=\gamma_{1}\left[\frac{d f_{0}}{d \phi}-(\Delta \phi+c|\nabla \phi|)\right],
$$

where $c$ is the curvature of interface. In the sharp-interface limit, the new term cancels the diffusion flux incurred by the Allen-Cahn dynamics, thus mass is conserved. On the downside, the ad hoc term prevents interfacial tension to be incorporated into the momentum equation via the phase field. Instead, it has to be added "by hand" through a spread-out delta function as in level-set and VOF methods.

Finally, we must point out that the phase-field dynamics do play a central role in a special class of two-phase flow problems where the interface undergoes topological changes such as breakup or coalescence [42]. The length scale of such critical processes approaches that of the interfacial thickness. In reality, these processes are dominated by short-range forces. As illustrated in the last section, the Cahn-Hilliard dynamics does contain a type of short-range force; it produces a disjoining pressure comparable to the van der Waals force. Then the question arises as to how closely this type of short-range force approximates reality in a particular experiment. The answer likely depends on the complex details of the experiment, as short-range forces from several sources can take part, typically imparting a stochasticity to the problem $[70,71]$. 
5.2. Interfacial relaxation. Secondary to the ambiguity in interfacial dynamics is the determination of parameter values. For the diffuse interface to reproduce the macroscopic behavior of a sharp-interface, the model parameters must be judiciously chosen. In particular, the parameter $\gamma_{1}$ determines the rate of relaxation of the $\phi$ field. However, there is little experimental information on $\gamma_{1}$ for the thin-interface two-phase flows that we are interested in. Jacqmin [34] juxtaposed two considerations on this: "straining flows can thin or thicken an interface and this must be resisted by a high enough diffusion. On the other hand, too large a diffusion will overly damp the flow". We will discuss several manifestations of interfacial relaxation in the following.

One interesting effect of interfacial relaxation is the initial "contraction" of a drop in a quiescent fluid. As an initial condition, we impose the hyperbolic tangential $\phi$ profile at the interface (equation 4.2), with $\phi= \pm 1$ in the two bulk phases. On commencing the simulation, however, we notice a very small shift in $\phi$ such that the interface $\phi=0$ shrinks slightly, and $\phi$ deviates from \pm 1 slightly in the bulk (Fig. 3 ). The reason for this artificial shrinkage is that the initial $\phi$ field is not the equilibrium one that minimizes the total free energy in $2 \mathrm{D}$. Thus, the interface tends to shrink to reduce the mixing energy. Since $\int_{\Omega} \phi d \Omega$ is conserved by the Cahn-Hilliard equation with the zero-flux boundary condition (equation 5.2), the shrinking interface causes the bulk $\phi$ value to change slightly, incurring an energy penalty in the bulk energy $f_{0}$. The competition between the bulk and interfacial energies results in a slightly relaxed $\phi$ field that has a lower energy than our initial condition. For a circular drop of radius $r$, one can calculate the shift in the bulk value of $\phi$ analytically if $\epsilon / r \ll 1$ :

$$
\delta \phi=\sqrt{2} \epsilon / 6 r
$$

In general, such a formula will not be available. But one may always choose a sufficiently small $\epsilon$ so that the initial shift is insignificant to the accuracy of the results.

Another important consequence of interfacial relaxation is the change in apparent interfacial tension $[41,43]$. To simulate an experiment with two immiscible fluids, one chooses appropriate values for the mixing energy $\lambda$ 


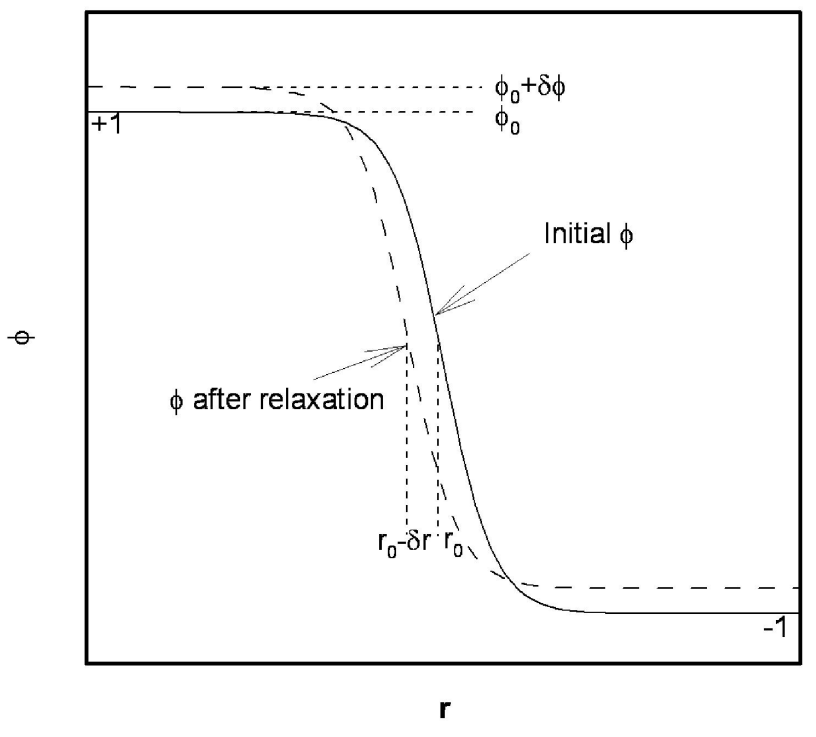

FIG. 3. A diagram showing the initial contraction of a drop in a quiescent matrix fluid.

and capillary width $\epsilon$ so as to match $\lambda / \epsilon$ to the experimental interfacial tension $\sigma$ according to a formula based on some equilibrium $\phi$ profile $[34,41]$. As $\phi$ evolves during flow, the matching formula no longer holds. Yue et al. [41] have shown an example of drop deformation in shear flows, where the deviation of the $\phi$ profile from the equilibrium one increases the effective interfacial tension. As a result, the drop deformation is underpredicted. Since the rate of relaxation is controlled by $\gamma_{1}$, it has an effect on the drop deformation as well. In this case, Fig. 4 shows that a smaller $\gamma_{1}$ increases the drop deformation slightly.

5.3. Interfacial thickness. The capillary width $\epsilon$ is another parameter that needs to be chosen carefully. This is a well-recognized issue in phase-field models for alloy solidification [29]. In our simulations of twophase flows, the interfacial thickness $h$, defined for example by $90 \%$ of the jump in $\phi$, is typically on the order of $4 \epsilon$. The smallest $h$ that one can resolve depends on the macroscopic length scale and the computational 


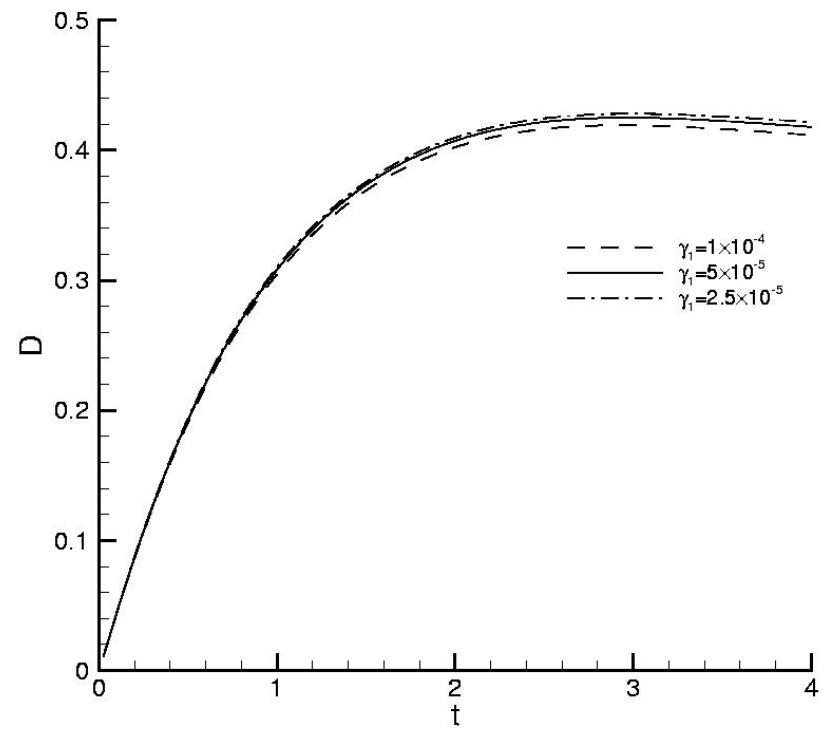

FIG. 4. Effect of the mobility parameter $\gamma_{1}$ on the deformation of a drop after startup of a simple shear. The drop is Newtonian while the matrix is a viscoelastic Oldroyd-B fluid. (after Yue et al. [41], (c) Cambridge University Press.)

capacity. But it is typically much thicker than the nano-scale real interfaces. Thus, it is a delicate task to pick an $\epsilon$ within one's computational reach that produces approximately the correct macroscopic behavior of a much thinner interface. As mentioned before, $\epsilon$ affects the effective interfacial tension, the relaxation of the interface and the short-range molecular forces. The philosophy behind choosing an appropriate value is perhaps best illustrated by a situation involving drastic topological changes.

Figures 5 and 6 show simulations with a larger or smaller $\epsilon$ than in Fig. 1 with all other parameters unchanged. The early stage of the simulations, say for $t \leq 1.342$, is identical with Fig. 1 . This is before the interfacial profiles of the two drops overlap. For a larger $\epsilon$, the interfaces of the two drops overlap at an earlier time during their approach, and the ensuing coalescence occurs more readily (Fig. 5). Note that the interface does not have time to develop the dimpled shape, and no matrix fluid is trapped inside the drop. On the other hand, a smaller $\epsilon$ prolongs the coalescence process 

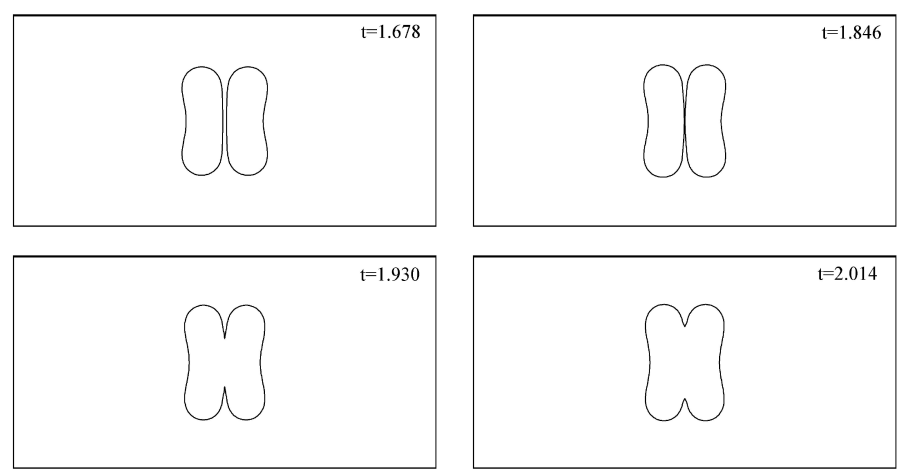

FIG. 5. Collision and coalescence of two Newtonian drops in a Newtonian matrix with a thicker interface. The parameters are the same as Fig. 1 except for $\epsilon=0.02$. (after Yue et al. [42], (C)Elsevier.)
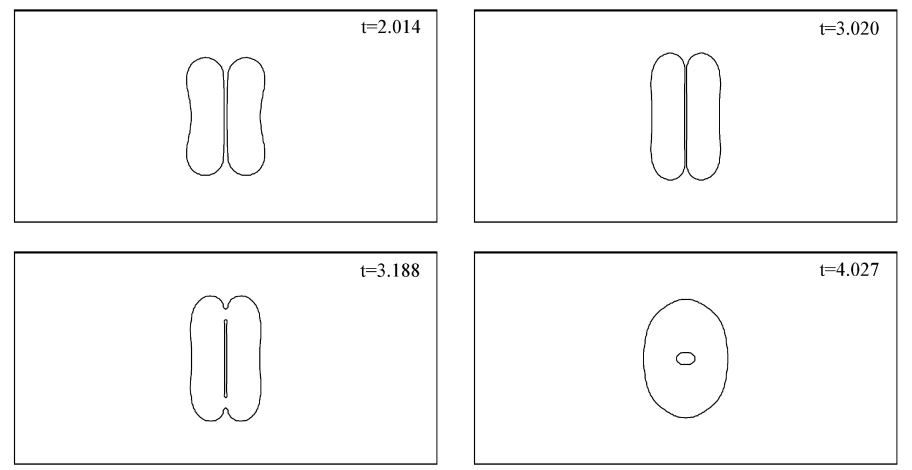

FIG. 6. Collision and coalescence of two Newtonian drops in a Newtonian matrix with a thinner interface. The parameters are the same as Fig. 1 except for $\epsilon=0.005$. (after Yue et al. [42], (C) Elsevier.)

(Fig. 6). As compared with Fig. 1, the points of rupture are more toward the ends of the film. This produces a less pronounced waist in the resultant compound drop, and the entrapped matrix filament does not break up but retracts into a droplet. The optimal $\epsilon$ cannot be determined by an a priori criterion. Rather, it needs to reflect the range of the molecular forces at work in the particular experiment to be simulated. Owing to a degree of randomness in the short-range forces, the coalescence time in experiments often exhibits a Gaussian distribution [70,71]. Obviously, such intricate details cannot be reproduced by the disjoining pressure in a phase-field 
formulation. Instead, one may hope to capture the macroscopic dynamics in some average sense by using optimal values for the model parameters.

Note that the effect of $\epsilon$ is not to be confused with numerical resolution of the interface. For each $\epsilon$ value tested here, mesh refinement has confirmed that the grid used is adequate for resolving the interface (see also [41]).

5.4. Adaptive mesh refinement. We argue that adaptive mesh refinement is capable of addressing all aforementioned issues. As has been established before, the diffuse-interface model will stay close to the sharpinterface model, with the conventional interfacial tension, when the interfacial thickness tends to zero $[33,37]$. Note that the $\phi$ profile as a solution to equation (2.6) is "nontrivial" only within the interfacial layer, whose thickness scales with $\epsilon$. Therefore, for sufficiently small transition thickness $\epsilon$ and elastic relaxation time $\gamma$, the effect of interfacial relaxation becomes negligible, and the difference between Cahn-Hilliard and Allen-Cahn dynamics becomes irrelevant. In fact, they represent two different regularizations of the kinematic transport of the phase field.

However, in some cases, such as those involving surfactant monolayers, the interfacial profile needs to be numerically resolved for accurate evaluation of the interfacial stress. The disparity between small $\epsilon$ and the global length scale implies the need for a locally refined grid inside the interfacial region.

Although procedures for dynamically adaptive meshing seem to be available [72,73], they have not been used in a diffuse-interface framework as we are aware. So far, we have used spectral methods with structured grids; the resolution of the interface is the numerical bottleneck [41] that must be tackled before the method can be used for large-scale flow simulations in three dimensions. Such an adaptive meshing scheme seems to be most conveniently implemented within a finite-element formulation. In addition, moving-mesh schemes may serve the same purpose. Code development along both directions is underway, and will be reported in the near future. 
5.5. Topological control. So far we have considered it an advantage that the phase-field method automatically handles topological changes such as merging and rupture of interfaces. This is the case when the nature of the short-range forces are understood and more or less adequately represented by the phase-field dynamics [42]. In certain applications, however, this may become a liability [74]. For instance, surface-active agents greatly modify the behavior of interfaces, stabilizing drops in emulsions and bubbles in foams against coarsening [1]. Membranes may prevent vesicles in contact from coalescing. If one chooses to use a phase-field model in such situations, it is desirable to retain some control of the topological events within the model. This consists of retrieving topological information from the phase field formulation, monitoring the occurrence of topological events, and even using the information to design a criterion for prohibiting unphysical changes of topology.

Du et al. [75] have recently developed a method for topological control in a phase-field model via the Euler number. The idea, briefly outlined below, applies equally well to other simulation methods for free boundary and interface problems such as the level-set methods.

Given an oriented (regular) compact (i.e., without boundary) surface $\Gamma$, the well-known Gauss-Bonnet formula states that

$$
\int_{\Gamma} K d s=2 \pi \chi
$$

where $K=k_{1} k_{2}$ is the Gaussian curvature of the surface in $R^{3}$, ds is the area element and $\chi / 2$ in $3 \mathrm{D}$ or $\chi$ in $2 \mathrm{D}$ is the Euler number [76]. The number $\chi$ is a commonly used topological quantity. For some frequently encountered surfaces, we have $\chi=2$ for a sphere, $\chi=0$ for a torus and $\chi=-2$ for a torus with 2 holes. For $2 \mathrm{D}$ curves, $K$ is the curvature and $\chi=1$ for a circle.

Such a concept can be generalized to the cases involving singularities, as illustrated in Figure 7. For instance, in 2 dimensional cases, we will have that:

$$
2 \pi \eta=\int_{\Gamma} K d s+\sum_{i=1}^{n}\left(\pi-\alpha_{i}\right)=2 \pi \chi+\sum_{i=1}^{n}\left(\pi-\alpha_{i}\right),
$$




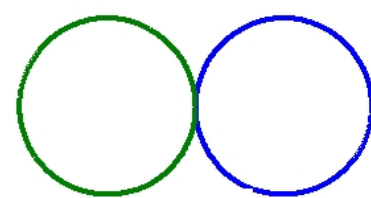

a

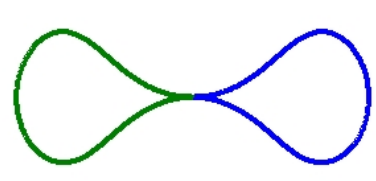

b

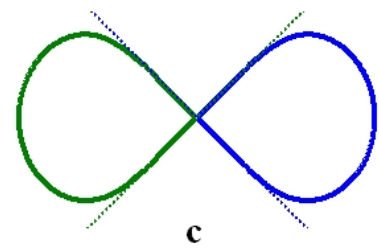

c

FIG. 7. Singular cases in 2-D. The inner intersect angles are $\pi, 0, \pi / 2$ for cases $a, b$ and $c$ respectively. The Euler number $\chi$ is 2, 1 and 1.5. The Euler-Poincaré index number $\eta$ is always 2.

where $\alpha_{i}$ are the inner angle at each vertices. And $\eta$, the Euler-Poincaré index number, is the topological integer, the genus of the surface.

In [75], we derived a phase-field representation of $\chi$. Let $\Gamma$ be a smooth oriented compact surface of a domain $\Omega$ in $\mathbf{R}^{3}$ (note that $\Gamma$ is allowed to have multiple disconnected pieces). Let $p$ be a monotonically increasing function defined from $\mathbf{R}$ to $\mathbf{R}$ with $p(0)=0$. We define the phase-field function as $\phi(x)=p(d(x))$ where the signed distance function $d(x)=$ $\operatorname{dist}(x, \Gamma)$ is defined to be positive inside $\Omega$ and negative outside $\Omega$. The level sets of $\phi$ are denoted by $\Gamma_{\mu}=\{x \in \Omega \mid \phi(x)=\mu\}$. In particular, we have $\Gamma=\Gamma_{0}$. We also define $\Omega^{\prime}=\{x \in \Omega \mid b<\phi(x)<a\}$, which forms a banded (layered) neighborhood around the surface for $b<0<a$. Further define $\Lambda(M)=\lambda_{1}(M) \lambda_{2}(M)=\Lambda\left(\nabla^{2} d(x)\right)$ for a singular matrix $M$ with $\lambda_{1}, \lambda_{2}$ being the two non-zero eigenvalues of $M=\nabla^{2} d(x)$. Since we can view that $k_{1}, k_{2}$ remain close to constant along the normal directions in the thin layer region $\Omega^{\prime}$, we have that

$$
\begin{aligned}
\frac{\chi}{2} & =\frac{1}{4 \pi} \int_{\Gamma} k_{1}(x) k_{2}(x) d s \\
& =\frac{1}{4 \pi(a-b)} \int_{p^{-1}(b)}^{p^{-1}(a)} p^{\prime}(\tau) d \tau \int_{\Gamma} k_{1}(x) k_{2}(x) d s \\
& =\frac{1}{4 \pi(a-b)} \int_{\Omega(a, b)} p^{\prime}(d(x)) k_{1}(x) k_{2}(x) d x \\
& =\frac{1}{4 \pi(a-b)} \int_{\Omega(a, b)} p^{\prime}(d(x)) \Lambda\left(\nabla^{2} d(x)\right) d x \\
& =\frac{1}{4 \pi(a-b)} \int_{\Omega(a, b)} \frac{1}{p^{\prime}(d(x))} \Lambda\left(\nabla^{2} \phi-p^{\prime \prime} \nabla_{i} d \nabla_{j} d\right) d x .
\end{aligned}
$$


In practice, the function $p$ and the constants $a, b$ will be chosen such that $p^{\prime}$ is relatively small outside of the transition layer. Now, since $p(x)$ is monotone, hence we have that $p^{\prime}(d(x))=|\nabla \phi(x)|$ and $p^{\prime \prime}(d(x))=\frac{\nabla|\nabla \phi|^{2} \cdot \nabla \phi}{2|\nabla \phi|^{2}}$. In the end we have the following theorem [75]:

Theorem 5.1. If $\phi=\phi(x)$ of $\Omega$ as $\phi(x)=p(d(x))$ where the signed distance function $d(x)=\operatorname{dist}(x, \Gamma)$. For any monotone increasing function $p$, there exists $b<0<a$, such that the following matrix $M$, where

$$
M(x)_{i j}=\frac{1}{2 \sqrt{\pi(a-b)|\nabla \phi|}}\left(\nabla_{i} \nabla_{j} \phi-\frac{\nabla|\nabla \phi|^{2} \cdot \nabla \phi}{2|\nabla \phi|^{4}} \nabla_{i} \phi \nabla_{j} \phi\right),
$$

is a singular matrix for $\forall x \in \Omega(a, b)$ in the sense that it always has a zero eigenvalue, and the Euler number of $\Gamma$ can be obtained as:

$$
\frac{\chi}{2}=\int_{\Omega(a, b)} F(x) d x
$$

where $F$ denote the coefficient of the linear term of the characteristic polynomial of $M$.

Numerical simulations, such as that in Figure 8 and Figure 9, show that the Euler number thus computed indeed captures the occurrence of critical topological events [75].
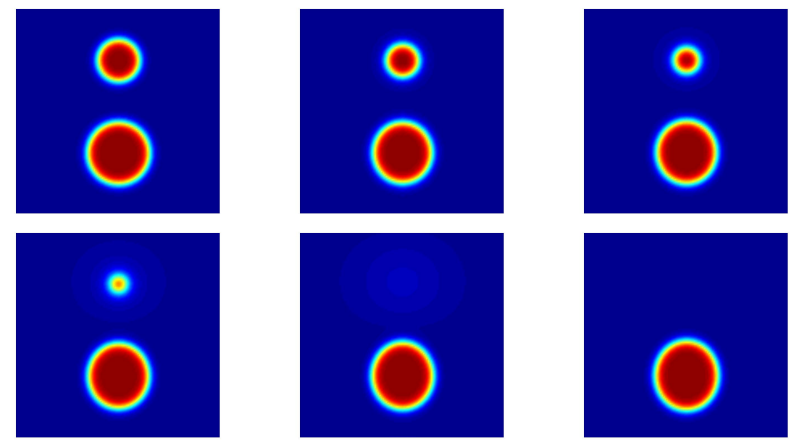

FIG. 8. Coalescence of two bubbles in a Newtonian fluid with the time valued at 0.00, 0.10, 0.18, 0.22, 0.24, 0.28. (after Du et al. [75].)

Besides detecting the occurrence of critical topological events, this quantity also provides an important tool in designing a scheme to prevent topological changes from happening. For instance, one may use a Lagrange multiplier to enforce the constancy of the Euler number over the entire domain. Since the constraint will involve a cost functional of high derivatives, more detailed analysis and numerical studies are needed in this area. 


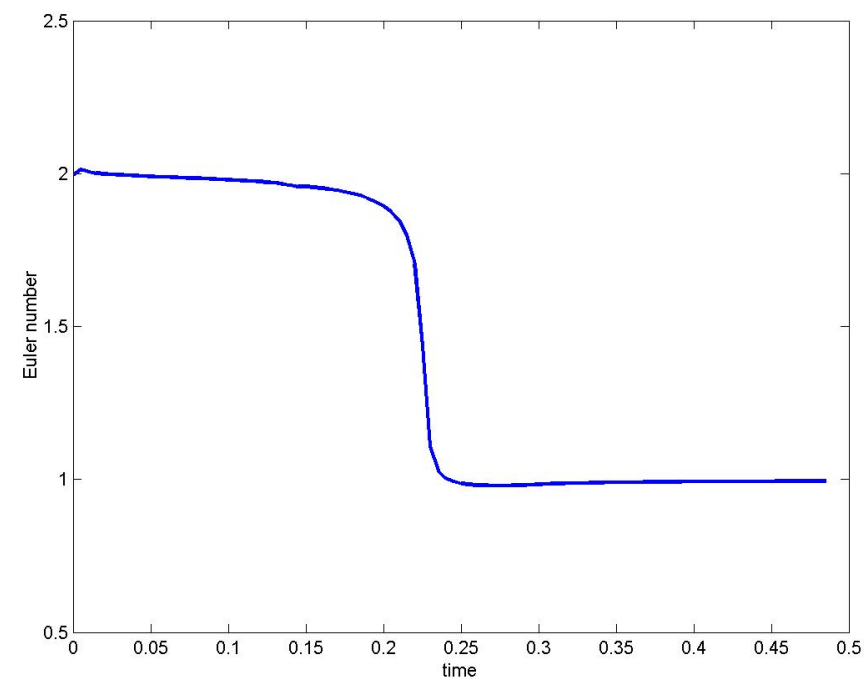

FIG. 9. A plot of the Euler number in time with the annihilation of the small bubble. (after Du et al. [75].)

6. Concluding remarks. This article aims to introduce the energetic variation based phase-field approach to readers interested in the fluid dynamics of immiscible complex fluids. Although various versions of the model have been used in the past to great degrees of success, we highlight the generic advantages inherent in the formalism. More importantly, perhaps, we discuss several detailed key crucial issues (challenges) with the method whose proper treatment is prerequisite to a physically realistic and numerically practicable implementation of the model.

We emphasize that the diffuse-interface treatment can alternatively be seen as a physical model or a numerical device. It can be viewed as a physics motivated approximation (regularization) of the sharp interface models. The employment of the phase field method changes the Lagrangian description of the interface motion into Eulerian description. The energetic variational procedure ensures that the resulting coupling system will still preserve the overall energy law. The method seems to be more appropriate for the drop dynamics problems that we have simulated, although there are other applications where the opposite is true. As such, the interfacial 
dynamics and model parameters do not directly correspond to measurable quantities and their determination is a delicate matter. We have advocated the view that the criterion should be that the diffuse-interface model accurately predict the macroscopic dynamics of the two-phase system, including drastic changes of the interfacial morphology. Several numerical experiments are shown to illustrate these issues and how they can be resolved to a satisfactory degree of accuracy. The inherent ambiguity vanishes as the interfacial thickness shrinks. Thus, we suggest adaptive mesh refinement as the solution when a thin interface has to be resolved. It is also necessary for computing large-scale 3D flows of blends of rheologically complex fluids.

7. Acknowledgments. Acknowledgment is made to the Donors of The Petroleum Research Fund, administered by the American Chemical Society, for partial support of this research. J.J.F. was also supported by the NSERC and the Canada Research Chairs program, and the NNSF of China (No. 20490220). C.L. was also supported by NSF grant DMS0405850. J.S. was partially supported by NSF grants DMS-0243191 and DMS-0311915. The authors also want to thank IMA for the support. This work was completed during the the workshop "Modeling of Soft Matter", for which the authors want to thank the organizers. The authors also want to thank Professors Q. Du, X. Feng and N. Walkington for many discussions over the years.

\section{REFERENCES}

[1] R. G. Larson. The Structure and Rheology of Complex Fluids. Oxford, New York, 1999.

[2] J. L. West. Polymer-dispersed liquid crystals. In R. A. Weiss and C. K. Ober, editors, Liquid-Crystalline Polymers, , R. A. Weiss and C. K. Ober editors, volume 435 of ACS Symp. Ser., chapter 32, pages 475-495. ACS, Washington, D.C., 1990.

[3] C. L. Tucker and P. Moldenaers. Microstructural evolution in polymer blends. Ann. Rev. Fluid Mech., 34:177-210, 2002.

[4] J. A. Sethian and P. Smereka. Level set methods for fluid interfaces. Ann. Rev. Fluid Mech., 35:341-372, 2003.

[5] J. Feng, G. Sgalari, and L. G. Leal. A theory for flowing nematic polymers with orientational distortion. J. Rheol., 44:1085-1101, 2000.

[6] V. Cristini, J. Blawzdziewicz, and M. Loewenberg. Drop breakup in threedimensional viscous flows. Phys. Fluids, 10:1781-1783, 1998.

[7] E. M. Toose, B. J. Geurts, and J. G. M. Kuerten. A boundary integral method for two-dimensional (non)-Newtonian drops in slow viscous flow. J. NonNewtonian Fluid Mech., 60:129-154, 1995. 
[8] R. E. Khayat. Three-dimensional boundary-element analysis of drop deformation for Newtonian and viscoelastic systems. Int. J. Num. Meth. Fluids, 34:241$275,2000$.

[9] H. H. Hu, N. A. Patankar, and M. Y. Zhu. Direct numerical simulations of fluidsolid systems using the arbitrary lagrangian-eulerian technique. J. Comput. Phys., 169:427-462, 2001.

[10] B. Ambravaneswaran, E. D. Wilkes, and O. A. Basaran. Drop formation from a capillary tube: Comparison of one-dimensional and two-dimensional analyses and occurrence of satellite drops. Phys. Fluids, 14:2606-2621, 2002.

[11] R. W. Hooper, V. F. de Almeida, C. W. Macosko, and J. J. Derby. Transient polymeric drop extension and retraction in uniaxial extensional flows. J. NonNewtonian Fluid Mech., 98:141-168, 2001.

[12] S. Ramaswamy and L. G. Leal. The deformation of a viscoelastic drop subjected to steady uniaxial extensional flow of a Newtonian fluid. J. Non-Newtonian Fluid Mech., 85:127-163, 1999.

[13] S. Ramaswamy and L. G. Leal. The deformation of a Newtonian drop in the uniaxial extensional flow of a viscoelastic liquid. J. Non-Newtonian Fluid Mech., 88:149-172, 1999.

[14] J. Li and Y. Renardy. Numerical study of flows of two immiscible liquids at low Reynolds number. SIAM Review, 42:417-439, 2000.

[15] Y. Renardy, M. Renardy, T. Chinyoka, D. B. Khismatullin, and J. Li. A viscoelastic vof-prost code for the study of drop deformation. Proc. 2004 ASME Heat Transfer/Fluids Engineering Summer Conference, ASME, HTFED2004-56114, 2004.

[16] J. Glimm, C. Klingenberg, O. McBryan, B. Plohr, D. Sharp, and S. Yaniv. Front tracking and two-dimensional Riemann problems. Adv. Appl. Math., 6:259290, 1985.

[17] S. O. Unverdi and G. Tryggvason. A front-tracking method for viscous, incompressible, multi-fluid flows. J. Comput. Phys., 100:25-37, 1992.

[18] Y. C. Chang, T. Y. Hou, B. Merriman, and S. Osher. A level set formulation of eulerian interface capturing methods for incompressible fluid flows. J. Comput. Phys., 124:449-464, 1996.

[19] J. A. Sethian. Level Set Methods and Fast Marching Methods: evolving interfaces in computational geometry, fluid mechanics, computer vision, and materials science, 2nd edition. Cambridge University Press, New York, 1999.

[20] S. Osher and R. Fedkiw. Level set methods: An overview and some recent results. J. Comput. Phys., 169:463-502, 2001.

[21] J. Lowengrub and L. Truskinovsky. Quasi-incompressible Cahn-Hilliard fluids and topological transitions. Proc. Roy. Soc. Lond. A, 454:2617-2654, 1998.

[22] J. D. van der Waals. The thermodynamic theory of capillarity under the hypothesis of a continuous variation of density. Verhandel Konink. Akad. Weten. Amsterdam, (Sect. 1), 1:1-56, 1892.

[23] J. D. van der Waals. The thermodynamic theory of capillarity under the hypothesis of a continuous variation of density, translation by j. s. rowlingson. J. Statist. Phys, 20:197-244, 1979.

[24] J. Dunn and J. Serrin. On the thermomechanics of interstitial working. Arch. Rational Mech. Anal., 88(2):95-133, 1985.

[25] D. Joseph. Fluid dynamics of two miscible liquids with diffusion and gradient stresses. European J. Mech. B Fluids, 9(6):565-596, 1990.

[26] P. C. Hohenberg and B. I. Halperin. Theory of dynamic critical phenomena. Rev. Mod. Phys., 49:435-479, 1977.

[27] A. J. Bray. Theory of phase-ording kinetics. Advances in Physics, 51(2):481-587, 2002.

[28] J. A. Warren and W. J. Boettinger. Prediction of dendritic growth and microsegregation patterns in a binary alloy using the phase-field method. Acta Metall. Mater., 43:689-703, 1995. 
[29] A. Karma. Phase-field formulation for quantitative modeling of alloy solidification. Phys. Rev. Lett., 87:115701, 2001.

[30] C. I. Poser and I. C. Sanchez. Interfacial tension theory of low and high molecular weight liquid mixtures. Macromolecules, 14:361-370, 1981.

[31] J. W. Cahn and J. E. Hilliard. Free energy of a nonuniform system. III. nucleation in a two-component incompressible fluid. J. Chem. Phys., 31:688-699, 1959.

[32] A. M. Lape na, S. C. Glotzer, S. A. Langer, and A. J. Liu. Effect of ordering on spinodal decomposition of liquid-crystal/polymer mixtures. Phys. Rev. E, 60:R29-R32, 1999.

[33] D. M. Anderson, G. B. McFadden, and A. A. Wheeler. Diffuse-interface methods in fluid mechanics. Ann. Rev. Fluid Mech., 30:139-165, 1998.

[34] D. Jacqmin. Calculation of two-phase Navier-Stokes flows using phase-field modelling. J. Comput. Phys., 155:96-127, 1999.

[35] M. Verschueren, F. N. van de Vosse, and H. E. H. Meijer. Diffuse-interface modelling of thermocapillary flow instabilities in a hele-shaw cell. J. Fluid Mech., 434:153-166, 2001

[36] V. E. Badalassi, H. D. Ceniceros, and S. Banerjee. Computation of multiphase systems with phase field model. J. Comput. Phys., 190:371-397, 2003.

[37] C. Liu and J. Shen. A phase field model for the mixture of two incompressible fluids and its approximation by a Fourier-spectral method. Physica D, 179:211-228, 2003.

[38] F. Boyer. A theoretical and numerical model for the study of incompressible mixture flows. Comput. Fluids, 31:41-68, 2002.

[39] T. Qian, X.-P. Wang, and P. Sheng. Generalized navier boundary condition for the moving contact line. Commun. Math. Sci., 1(2):333-341, 2003.

[40] M. Gurtin, D. Polignone, and J. Vinals. Two-phase binary fluids and immiscible fluids described by an order parameter. Math. Models Methods Appl. Sci., 6(6):815-831, 1996.

[41] P. Yue, J. J. Feng, C. Liu, and J. Shen. A diffuse-interface method for simulating two-phase flows of complex fluids. J. Fluid Mech., 515:293-317, 2004.

[42] P. Yue, J. J. Feng, C. Liu, and J. Shen. Diffuse-interface simulations of drop coalescence and retraction in viscoelastic fluids. J. Non-Newtonian Fluid Mech., accepted, 2005.

[43] P. Yue, J. J. Feng, C. Liu, and J. Shen. Interfacial force and Marangoni flow on a nematic drop retracting in an isotropic fluid. J. Colloid Interface Sci., accepted, 2005.

[44] P. Yue, J. J. Feng, C. Liu, and J. Shen. Viscoelastic effects on drop deformation in steady shear. J. Fluid Mech., submitted, 2004.

[45] P. Yue, J. J. Feng, C. Liu, and J. Shen. Transient drop deformation upon startup of shear in viscoelastic fluids. Phys. Fluids, submitte, 2005.

[46] P. Yue, J. J. Feng, C. Liu, and J. Shen. Heart-shaped bubbles rising in a nematic fluid. in preparation, 2005.

[47] S. R. de Groot and P. Mazur. Nonequilibrium Thermodynamics. North Holland, 1962.

[48] C. Liu, J. Shen, J. J. Feng, and P. Yue. Variational approach in two-phase flows of complex fluids: transport and induced elastic stress. In A. Miranville, editor, Mathematical Models and Methods in Phase Transitions,. Nova Publications, 2005.

[49] C. Liu and N. J. Walkington. An eulerian description of fluids containing viscoelastic particles. Arch. Ration. Mech. Anal., 159(3):229-252, 2001.

[50] P. G. de Gennes and J. Prost. The Physics of Liquid Crystals. Oxford, New York, 1993.

[51] C. Liu and N. J. Walkington. Approximation of liquid crystal flows. SIAM J. Numer. Anal., 37:725-741, 2000.

[52] A. Rapini and M. Popoular. Distortion d'une lamelle nematique sous champ magnetique conditions d'ancrage aux parois. J. Phys. (Paris) C, 30:54-56, 1969. 
[53] C. Liu and J. Shen. On liquid crystal flows with free-slip boundary conditions. Dis. Cont. Dyn. Sys., 7:307-318, 2001.

[54] J. Shen. Efficient spectral-Galerkin method. II. direct solvers of second and fourth order equations by using Chebyshev polynomials. SIAM J. Sci. Comput., 16:74-87, 1995.

[55] J. L. Guermond, P. Minev, and J. Shen. An overview of projection methods for incompressible flows. Comput. Methods Appl. Mech. Eng., submitted, 2005.

[56] J. L. Guermond and J. Shen. A class of truly consistent splitting schemes for incompressible flows. J. Comput. Phys., 192:262-276, 2003.

[57] J. Shen. Efficient spectral-Galerkin method. I. direct solvers of second and fourth order equations by using Legendre polynomials. SIAM J. Sci. Comput., 15:1489-1505, 1994.

[58] J. Shen. Efficient spectral-Galerkin method. III. polar and cylindrical geometries. SIAM J. Sci. Comput., 18:1583-160, 1997.

[59] X. Zhang, R. S. Padgett, and O. A. Basaran. Nonlinear deformation and breakup of stretching liquid bridges. J. Fluid Mech., 329:207-245, 1996.

[60] M. R. Nobari, Y.-J. Jan, and G. Tryggvason. Head-on collision of drops-a numerical investigation. Phys. Fluids, 8:29-42, 1996.

[61] A. N. Zdravkov, G. W. M. Peters, and H. E. H. Meijer. Film drainage between two captive drops: PEO-water in silicon oil. J. Colloid Interface Sci., 266:195-201, 2003.

[62] A. Bhakta and E. Ruckenstein. Decay of standing foams: drainage, coalescence and collapse. Adv. Colloid Interface Sci., 70:1-124, 1997.

[63] L. M. Pismen. Nonlocal diffuse interface theory of thin films and the moving contact line. Phys. Rev. E, 64:021603, 2001.

[64] R. B. Bird, D. F. Curtiss, R. C. Armstrong, and O. Hassager. Dynamics of Polymeric Liquids, Vol. 2. Kinetic Theory. Wiley, New York, 1987.

[65] G. Marrucci and F. Greco. The elastic constants of Maier-Saupe rodlike molecule nematics. Mol. Cryst. Liq. Cryst., 206:17-30, 1991.

[66] F. H. Lin and C. Liu. Nonparabolic dissipative systems, modeling the flow of liquid crystals. Comm. Pure Appl. Math., 48:501-537, 1995.

[67] F. H. Lin and C. Liu. Existence of solutions for the Ericksen-Leslie system. Arch. Rat. Mech. Anal., 154:135-156, 2000.

[68] X. Yang, J. J. Feng, C. Liu, and J. Shen. Contraction and pinch-off phenomena of a liquid filament. J. Comput. Phys., submitted, 2005.

[69] T. Biben, C. Misbah, A. Leyrat, and C. Verdier. An advected-field approach to the dynamics of fluid interfaces. Europhys. Lett., 63:623-629, 2003.

[70] G. E. Charles and S. G. Mason. The coalescence of liquid drops with flat liquidliquid interfaces. J. Colloid Sci., 15:236-267, 1960.

[71] P. Ghosh and V. A. Juvekar. Analysis of the drop rest phenomenon. Chem. Eng. Res. Design, 80:715-728, 2002.

[72] C. F. Ollivier-Gooch. Coarsening unstructured meshes by edge contraction. Int. J. Numer. Methods Eng., 57:391-414, 2003.

[73] V. Cristini and Y. C. Tan. Theory and numerical simulation of droplet dynamics in complex flows - a review. Lab Chip, 4:257-264, 2004.

[74] M. van Sint Annaland, N. G. Deen, and J. A. M. Kuipers. Numerical simulation of gas bubbles behaviour using a three-dimensional volume of fluid method. Chem. Eng. Sci., 60:2999-3011, 2005.

[75] Q. Du, C. Liu, and X. Wang. Retrieving topological information for phase field models. SIAM J. Appl. Math., accepted for publication, 2005.

[76] M. P. do Carmo. Differential Geometry of Curves and Surfaces. Prentice-Hall, Englewood Cliffs, NJ, 1976. 